\title{
Fixed points and approximately heptic mappings in non-Archimedean normed spaces
}

\author{
Choonkil Park', Mohammad Bagher Ghaemi ${ }^{1}$, Hamid Majani², Madjid Eshaghi Gordji ${ }^{3 *}$ and Abbas Najati ${ }^{4}$
}

"Correspondence:

madjid.eshaghi@gmail.com

${ }^{3}$ Department of Mathematics, Semnan University, Semnan, Iran

Full list of author information is

available at the end of the article

\begin{abstract}
Using the fixed point method, we investigate the stability of the system of additive, quadratic and quartic functional equations with constant coefficients in non-Archimedean normed spaces. Also, we give an example to show that some results in the stability of functional equations in (Archimedean) normed spaces are not valid in non-Archimedean normed spaces.
\end{abstract}

MSC: 39B82; 46S10;47H10

Keywords: heptic functional equation; Hyers-Ulam stability; fixed point method

\section{Introduction}

The stability problems concerning group homomorphisms was raised by Ulam [1] in 1940 and affirmatively answered for Banach spaces by Hyers [2] in the next year. Hyers' theorem was generalized by Aoki [3] for additive mappings and by Rassias [4] for linear mappings by considering an unbounded Cauchy difference. In 1994, a generalization of the Rassias theorem was obtained by Găvruta [5] by replacing the unbounded Cauchy difference by a general control function.

In 2003, Radu [6] proposed a new method for obtaining the existence of exact solutions and error estimations, based on the fixed point alternative (see also $[7,8]$ ).

Let $(X, d)$ be a generalized metric space. An operator $T: X \rightarrow X$ satisfies a Lipschitz condition with the Lipschitz constant $L$ if there exists a constant $L \geq 0$ such that $d(T x, T y) \leq$ $L d(x, y)$ for all $x, y \in X$. If the Lipschitz constant $L$ is less than 1 , then the operator $T$ is called a strictly contractive operator. Note that the distinction between the generalized metric and the usual metric is that the range of the former is permitted to include the infinity. We recall the following theorem by Margolis and Diaz.

Theorem $1.1(c f .[6,9])$ Suppose that we are given a complete generalized metric space $(\Omega, d)$ and a strictly contractive mapping $T: \Omega \rightarrow \Omega$ with the Lipschitz constant L. Then, for each given $x \in \Omega$, either

$$
d\left(T^{m} x, T^{m+1} x\right)=\infty \text { for all } m \geq 0,
$$

or there exists a natural number $m_{0}$ such that

$$
\text { - } d\left(T^{m} x, T^{m+1} x\right)<\infty \text { for all } m \geq m_{0}
$$

O 2013 Park et al.; licensee Springer. This is an Open Access article distributed under the terms of the Creative Commons Attribution License (http://creativecommons.org/licenses/by/2.0), which permits unrestricted use, distribution, and reproduction in any medium, provided the original work is properly cited. 
- the sequence $\left\{T^{m} x\right\}$ is convergent to a fixed point $y^{\prime \prime}$ of $T$;

- $y^{\prime \prime}$ is the unique fixed point of $T$ in $\Lambda=\left\{y \in \Omega: d\left(T^{m_{0}} x, y\right)<\infty\right\}$;

- $d\left(y, y^{*}\right) \leq \frac{1}{1-L} d(y, T y)$ for all $y \in \Lambda$.

Hensel [10] has introduced a normed space which does not have the Archimedean property. During the last three decades, the theory of non-Archimedean spaces has gained the interest of physicists for their research, in particular, in problems coming from quantum physics, $p$-adic strings and superstrings [11]. Although many results in the classical normed space theory have a non-Archimedean counterpart, their proofs are different and require a rather new kind of intuition [12-15].

One may note that $|n| \leq 1$ in each valuation field, every triangle is isosceles and there may be no unit vector in a non-Archimedean normed space; $c f$. [13]. These facts show that the non-Archimedean framework is of special interest.

Definition 1.2 Let $\mathbb{K}$ be a field. A valuation mapping on $\mathbb{K}$ is a function $|\cdot|: \mathbb{K} \rightarrow \mathbb{R}$ such that for any $a, b \in \mathbb{K}$ we have

(i) $|a| \geq 0$ and equality holds if and only if $a=0$,

(ii) $|a b|=|a||b|$,

(iii) $|a+b| \leq|a|+|b|$.

A field endowed with a valuation mapping will be called a valued field. If the condition (iii) in the definition of a valuation mapping is replaced with

(iii) $^{\prime}|a+b| \leq \max \{|a|,|b|\}$,

then the valuation $|\cdot|$ is said to be non-Archimedean. The condition (iii) ${ }^{\prime}$ is called the strict triangle inequality. By (ii), we have $|1|=|-1|=1$. Thus, by induction, it follows from (iii) $^{\prime}$ that $|n| \leq 1$ for each integer $n$. We always assume in addition that $|\cdot|$ is nontrivial, i.e., that there is an $a_{0} \in \mathbb{K}$ such that $\left|a_{0}\right| \notin\{0,1\}$. The most important examples of nonArchimedean spaces are $p$-adic numbers.

Definition 1.3 Let $X$ be a linear space over a scalar field $\mathbb{K}$ with a non-Archimedean nontrivial valuation $|\cdot|$. A function $\|\cdot\|: X \rightarrow \mathbb{R}$ is a non-Archimedean norm (valuation) if it satisfies the following conditions:

(NA1) $\|x\|=0$ if and only if $x=0$;

(NA2) $\|r x\|=|r|\|x\|$ for all $r \in \mathbb{K}$ and $x \in X$;

(NA3) the strong triangle inequality (ultrametric); namely

$$
\|x+y\| \leq \max \{\|x\|,\|y\|\} \quad(x, y \in X) .
$$

Then $(X,\|\cdot\|)$ is called a non-Archimedean normed space.

It follows from (NA3) that

$$
\left\|x_{m}-x_{l}\right\| \leq \max \left\{\left\|x_{J+1}-x_{J}\right\|: l \leq J \leq m-1\right\} \quad(m>l),
$$

and so a sequence $\left\{x_{m}\right\}$ is Cauchy in $X$ if and only if $\left\{x_{m+1}-x_{m}\right\}$ converges to zero in a non-Archimedean space. It is easy to see that every convergent sequence in a non- 
Archimedean normed space is Cauchy. If each Cauchy sequence is convergent, then the non-Archimedean normed space is said to be complete and is called a non-Archimedean Banach space.

Khodaei and Rassias [16] investigated the solution and stability of the $n$-dimensional additive functional equation such that in the special case $n=2$,

$$
f(a x+b y)+f(a x-b y)=2 a f(x)
$$

for $a, b \in \mathbb{Z} \backslash\{0\}$ with $a \neq \pm 1, \pm b$. The authors proved that the Cauchy equation is equivalent to the above equation.

The functional equation

$$
f(x+y)+f(x-y)=2 f(x)+2 f(y)
$$

is related to a symmetric bi-additive function $[17,18]$. It is natural that this equation is called a quadratic functional equation. In particular, every solution of quadratic equation (1.2) is said to be a quadratic function. It is well known that a function $f$ between real vector spaces is quadratic if and only if there exists a unique symmetric bi-additive function $B_{1}$ such that $f(x)=B_{1}(x, x)$ for all $x$. The bi-additive function $B_{1}$ is given by $B_{1}(x, y)=\frac{1}{4}(f(x+$ $y)-f(x-y))$. The Hyers-Ulam stability problem for the quadratic functional equation was solved by Skof [19]. In [20], Czerwik proved the Hyers-Ulam stability of equation (1.2). Eshaghi Gordji and Khodaei [21] obtained the general solution and the Hyers-Ulam stability of the following quadratic functional equation for $a, b \in \mathbb{Z} \backslash\{0\}$ with $a \neq \pm 1, \pm b$ :

$$
f(a x+b y)+f(a x-b y)=2 a^{2} f(x)+2 b^{2} f(y) .
$$

Lee et al. [22] considered the following functional equation:

$$
f(2 x+y)+f(2 x-y)=4(f(x+y)+f(x-y))+24 f(x)-6 f(y) .
$$

In fact, they proved that a mapping $f$ between two real vector spaces $X$ and $Y$ is a solution of (1.4) if and only if there exists a unique symmetric bi-quadratic mapping $B_{2}: X \times X \rightarrow Y$ such that $f(x)=B_{2}(x, x)$ for all $x$. The bi-quadratic mapping $B_{2}$ is given by

$$
B_{2}(x, y)=\frac{1}{12}(f(x+y)+f(x-y)-2 f(x)-2 f(y)) .
$$

Obviously, the function $f(x)=a x^{4}$ satisfies functional equation (1.4), which is called a quartic functional equation. Kang [23] investigated the Hyers-Ulam stability problem for the quartic functional equation

$$
\begin{aligned}
& f(a x+b y)+f(a x-b y) \\
& \quad=a^{2} b^{2}(f(x+y)+f(x-y))+2 a^{2}\left(a^{2}-b^{2}\right) f(x)-2 b^{2}\left(a^{2}-b^{2}\right) f(y),
\end{aligned}
$$

where $a, b \in \mathbb{Z} \backslash\{0\}$ with $a \neq \pm 1, \pm b$. For other quartic functional equations, see [24-28]. For other functional equations, see [29-42]. 
Ebadian et al. [43] considered the Hyers-Ulam stability of the systems of additive-quartic functional equations

$$
\left\{\begin{array}{l}
f\left(x_{1}+x_{2}, y\right)=f\left(x_{1}, y\right)+f\left(x_{2}, y\right) \\
f\left(x, 2 y_{1}+y_{2}\right)+f\left(x, 2 y_{1}-y_{2}\right) \\
=4 f\left(x, y_{1}+y_{2}\right)+4 f\left(x, y_{1}-y_{2}\right)+24 f\left(x, y_{1}\right)-6 f\left(x, y_{2}\right)
\end{array}\right.
$$

and the quadratic-cubic functional equations

$$
\left\{\begin{array}{l}
f\left(x, 2 y_{1}+y_{2}\right)+f\left(x, 2 y_{1}-y_{2}\right)=2 f\left(x, y_{1}+y_{2}\right)+2 f\left(x, y_{1}-y_{2}\right)+12 f\left(x, y_{1}\right), \\
f\left(x, y_{1}+y_{2}\right)+f\left(x, y_{1}-y_{2}\right)=2 f\left(x, y_{1}\right)+2 f\left(x, y_{2}\right) .
\end{array}\right.
$$

In this paper, we investigate the Hyers-Ulam stability for the system of additive, quadratic and quartic functional equations

$$
\left\{\begin{array}{l}
f\left(a x_{1}+b x_{2}, y, z\right)+f\left(a x_{1}-b x_{2}, y, z\right)=2 a f\left(x_{1}, y, z\right) \\
f\left(x, a y_{1}+b y_{2}, z\right)+f\left(x, a y_{1}-b y_{2}, z\right)=2 a^{2} f\left(x, y_{1}, z\right)+2 b^{2} f\left(x, y_{2}, z\right), \\
f\left(x, y, a z_{1}+b z_{2}\right)+f\left(x, y, a z_{1}-b z_{2}\right) \\
\quad=a^{2} b^{2}\left(f\left(x, y, z_{1}+z_{2}\right)+f\left(x, y, z_{1}-z_{2}\right)\right) \\
\quad+2 a^{2}\left(a^{2}-b^{2}\right) f\left(x, y, z_{1}\right)-2 b^{2}\left(a^{2}-b^{2}\right) f\left(x, y, z_{2}\right)
\end{array}\right.
$$

where $a, b \in \mathbb{Z} \backslash\{0\}$ with $a \neq \pm 1, \pm b$. The function $f: \mathbb{R} \times \mathbb{R} \times \mathbb{R} \rightarrow \mathbb{R}$ given by $f(x, y, z)=$ $c x y^{2} z^{4}$ is a solution of (1.8). In particular, letting $y=z=x$, we get a heptic function $g: \mathbb{R} \rightarrow$ $\mathbb{R}$ in one variable given by $g(x):=f(x, x)=c x^{7}$. The proof of the following proposition is evident, and we omit the details.

Proposition 1.4 Let $X$ and $Y$ be real linear spaces. If a mapping $f: X \times X \times X \rightarrow Y$ satisfies system (1.8), then $f(\lambda x, \mu y, \eta z)=\lambda \mu^{2} \eta^{4} f(x, y, z)$ for all $x, y, z \in X$, and all rational numbers $\lambda, \mu, \eta$.

In the rest of this paper, unless otherwise explicitly stated, we assume that $X$ is a nonArchimedean normed space and $Y$ is a non-Archimedean Banach space.

\section{Approximation of heptic mappings}

In this section, we investigate the Hyers-Ulam stability problem for the system of functional equations (1.8) in non-Archimedean Banach spaces.

Theorem 2.1 Let $s \in\{-1,1\}$ be fixed. Let $\phi_{1}, \phi_{2}, \phi_{3}: X \times X \times X \times X \rightarrow[0, \infty)$ be functions such that

$$
\begin{aligned}
\Phi(x, y, z):= & \left|\frac{1}{2}\right| \max \left\{\left|a^{\frac{5-7 s}{2}}\right| \phi_{1}\left(a^{\frac{s-1}{2}} x, 0, a^{\frac{s-1}{2}} y, a^{\frac{s-1}{2}} z\right),\right. \\
& \left|a^{\frac{1-7 s}{2}}\right| \phi_{2}\left(a^{\frac{s+1}{2}} x, a^{\frac{s-1}{2}} y, 0, a^{\frac{s-1}{2}} z\right), \\
& \left.\left|a^{-\frac{7+7 s}{2}}\right| \phi_{3}\left(a^{\frac{s+1}{2}} x, a^{\frac{s+1}{2}} y, a^{\frac{s-1}{2}} z, 0\right)\right\}
\end{aligned}
$$


for all $x, y, z \in X$, and for some $0<L<1$,

$$
\Phi\left(a^{s} x, a^{s} y, a^{s} z\right) \leq L\left|a^{7 s}\right| \Phi(x, y, z)
$$

and

$$
\begin{aligned}
& \lim _{n \rightarrow \infty}\left|a^{-7 s n}\right| \phi_{1}\left(a^{s n} x_{1}, a^{s n} x_{2}, a^{s n} y, a^{s n} z\right)=0, \\
& \lim _{n \rightarrow \infty}\left|a^{-7 s n}\right| \phi_{2}\left(a^{s n} x, a^{s n} y_{1}, a^{s n} y_{2}, a^{s n} z\right)=0, \\
& \lim _{n \rightarrow \infty}\left|a^{-7 s n}\right| \phi_{3}\left(a^{s n} x, a^{s n} y, a^{s n} z_{1}, a^{s n} z_{2}\right)=0
\end{aligned}
$$

for all $x, y, z, x_{1}, x_{2}, y_{1}, y_{2}, z_{1}, z_{2} \in X$. If $: X \times X \times X \rightarrow Y$ is a mapping such that $f(x, 0, z)=$ $f(x, y, 0)=0$ for all $x, y, z \in X$, and

$$
\begin{aligned}
& \left\|f\left(a x_{1}+b x_{2}, y, z\right)+f\left(a x_{1}-b x_{2}, y, z\right)-2 a f\left(x_{1}, y, z\right)\right\| \leq \phi_{1}\left(x_{1}, x_{2}, y, z\right), \\
& \left\|f\left(x, a y_{1}+b y_{2}, z\right)+f\left(x, a y_{1}-b y_{2}, z\right)-2 a^{2} f\left(x, y_{1}, z\right)-2 b^{2} f\left(x, y_{2}, z\right)\right\| \\
& \quad \leq \phi_{2}\left(x, y_{1}, y_{2}, z\right), \\
& \| f\left(x, y, a z_{1}+b z_{2}\right)+f\left(x, y, a z_{1}-b z_{2}\right)-a^{2} b^{2}\left(f\left(x, y, z_{1}+z_{2}\right)+f\left(x, y, z_{1}-z_{2}\right)\right) \\
& \quad-2 a^{2}\left(a^{2}-b^{2}\right) f\left(x, y, z_{1}\right)+2 b^{2}\left(a^{2}-b^{2}\right) f\left(x, y, z_{2}\right) \| \leq \phi_{3}\left(x, y, z_{1}, z_{2}\right)
\end{aligned}
$$

for all $x, y, z, x_{1}, x_{2}, y_{1}, y_{2}, z_{1}, z_{2} \in X$, then there exists a unique heptic mapping $T: X \times X \times$ $X \rightarrow Y$ satisfying (1.8) and

$$
\|f(x, y, z)-T(x, y, z)\| \leq \frac{1}{1-L} \Phi(x, y, z)
$$

for all $x, y, z \in X$.

Proof Putting $x_{1}=2 x$ and $x_{2}=0$ and replacing $y, z$ by $2 y, 2 z$ in (2.3), we get

$$
\|f(2 a x, 2 y, 2 z)-a f(2 x, 2 y, 2 z)\| \leq\left|\frac{1}{2}\right| \phi_{1}(2 x, 0,2 y, 2 z)
$$

for all $x, y, z \in X$. Putting $y_{1}=2 y$ and $y_{2}=0$ and replacing $x, z$ by $2 a x, 2 z$ in (2.4), we get

$$
\left\|f(2 a x, 2 a y, 2 z)-a^{2} f(2 a x, 2 y, 2 z)\right\| \leq\left|\frac{1}{2}\right| \phi_{2}(2 a x, 2 y, 0,2 z)
$$

for all $x, y, z \in X$. Putting $z_{1}=2 z$ and $z_{2}=0$ and replacing $x, y$ by $2 a x$, 2ay in (2.5), we get

$$
\left\|f(2 a x, 2 a y, 2 a z)-a^{4} f(2 a x, 2 a y, 2 z)\right\| \leq\left|\frac{1}{2}\right| \phi_{3}(2 a x, 2 a y, 2 z, 0)
$$

for all $x, y, z \in X$. Thus

$$
\begin{aligned}
& \left\|f(2 a x, 2 a y, 2 a z)-a^{7} f(2 x, 2 y, 2 z)\right\| \\
& \quad \leq\left|\frac{1}{2}\right| \max \left\{\left|a^{6}\right| \phi_{1}(2 x, 0,2 y, 2 z),\left|a^{4}\right| \phi_{2}(2 a x, 2 y, 0,2 z), \phi_{3}(2 a x, 2 a y, 2 z, 0)\right\}
\end{aligned}
$$


for all $x, y, z \in X$. Replacing $x, y$ and $z$ by $\frac{x}{2}, \frac{y}{2}$ and $\frac{z}{2}$ in (2.10), we have

$$
\begin{aligned}
& \left\|f(a x, a y, a z)-a^{7} f(x, y, z)\right\| \\
& \quad \leq\left|\frac{1}{2}\right| \max \left\{\left|a^{6}\right| \phi_{1}(x, 0, y, z),\left|a^{4}\right| \phi_{2}(a x, y, 0, z), \phi_{3}(a x, a y, z, 0)\right\}
\end{aligned}
$$

for all $x, y, z \in X$. It follows from (2.11) that

$$
\begin{aligned}
& \left\|\frac{1}{a^{7}} f(a x, a y, a z)-f(x, y, z)\right\| \\
& \quad \leq\left|\frac{1}{2}\right| \max \left\{\left|a^{-1}\right| \phi_{1}(x, 0, y, z),\left|a^{-3}\right| \phi_{2}(a x, y, 0, z),\left|a^{-7}\right| \phi_{3}(a x, a y, z, 0)\right\}, \\
& \left\|a^{7} f\left(\frac{x}{a}, \frac{y}{a}, \frac{z}{a}\right)-f(x, y, z)\right\| \\
& \quad \leq\left|\frac{1}{2}\right| \max \left\{\left|a^{6}\right| \phi_{1}\left(\frac{x}{a}, 0, \frac{y}{a}, \frac{z}{a}\right),\left|a^{4}\right| \phi_{2}\left(x, \frac{y}{a}, 0, \frac{z}{a}\right), \phi_{3}\left(x, y, \frac{z}{a}, 0\right)\right\}
\end{aligned}
$$

for all $x, y, z \in X$. From the inequalities (2.12) and (2.13), we obtain

$$
\left\|\frac{1}{a^{7 s}} f\left(a^{s} x, a^{s} y, a^{s} z\right)-f(x, y, z)\right\| \leq \Phi(x, y, z)
$$

for all $x, y, z \in X$.

Let $S$ be the set of all mappings $h: X \times X \times X \rightarrow Y$ with $h(x, 0, z)=h(x, y, 0)=0$ for all $x, y, z \in X$, and let us introduce a generalized metric on $S$ as follows:

$$
d(h, k)=\inf \left\{u \in \mathbb{R}^{+}:\|(h(x, y, z)-k(x, y, z))\| \leq u \Phi(x, y, z), \forall x, y, z \in X\right\}
$$

where, as usual, $\inf \emptyset=+\infty$. The proof of the fact that $(S, d)$ is a complete generalized metric space can be found in $[7,44]$. Now we consider the mapping $J: S \rightarrow S$ defined by

$$
\operatorname{Jh}(x, y):=a^{-7 s} h\left(a^{s} x, a^{s} y, a^{s} z\right)
$$

for all $h \in S$ and $x, y \in X$. Let $\varepsilon>0$ and $f, g \in S$ be such that $d(f, g)<\varepsilon$. Then

$$
\begin{aligned}
\|J f(x, y)-J g(x, y)\| & =\left\|a^{-7 s} f\left(a^{s} x, a^{s} y, a^{s} z\right)-a^{-7 s} g\left(a^{s} x, a^{s} y, a^{s} z\right)\right\| \\
& =\left|a^{-7 s}\right|\left\|f\left(a^{s} x, a^{s} y, a^{s} z\right)-g\left(a^{s} x, a^{s} y, a^{s} z\right)\right\| \\
& \leq\left|a^{-7 s}\right| \varepsilon \Phi\left(a^{s} x, a^{s} y, a^{s} z\right) \leq L \varepsilon \Phi(x, y, z),
\end{aligned}
$$

that is, if $d(f, g)<\varepsilon$, we have $d(J f, J g) \leq L \varepsilon$. This means that

$$
d(J f, J g) \leq L d(f, g)
$$

for all $f, g \in S$. So, $J$ is a strictly contractive self-mapping on $S$ with the Lipschitz constant $L$. It follows from $(2.14)$ that $d(J f, f) \leq 1$. Due to Theorem 1.1, there exists a unique mapping 
$T: X \times X \times X \rightarrow Y$ such that $T$ is a fixed point of $J$, i.e., $T\left(a^{s} x, a^{s} y, a^{s} z\right)=a^{7 s} T(x, y, z)$ for all $x, y, z \in X$. Also, $d\left(J^{m} f, T\right) \rightarrow 0$ as $m \rightarrow \infty$, which implies the equality

$$
\lim _{m \rightarrow \infty} a^{-7 s m} f\left(a^{s m} x, a^{s m} y, a^{s m} z\right)=T(x, y, z)
$$

for all $x, y, z \in X$.

It follows from (2.2), (2.3), (2.4) and (2.5) that

$$
\begin{aligned}
& \| T\left(a x_{1}+b x_{2}, y, z\right)+T\left(a x_{1}-b x_{2}, y, z\right)-2 a T\left(x_{1}, y, z\right) \| \\
&= \lim _{n \rightarrow \infty}\left|a^{-7 s n}\right| \| f\left(a^{s n} a x_{1}+a^{s n} b x_{2}, a^{s n} y, a^{s n} z\right)+f\left(a^{s n} a x_{1}-a^{s n} b x_{2}, a^{s n} y, a^{s n} z\right) \\
&-2 a f\left(a^{s n} x_{1}, a^{s n} y, a^{s n} z\right) \| \\
& \leq \lim _{n \rightarrow \infty}\left|a^{-7 s n}\right| \phi_{1}\left(a^{s n} x_{1}, a^{s n} x_{2}, a^{s n} y, a^{s n} z\right)=0, \\
&\left\|T\left(x, a y_{1}+b y_{2}, z\right)+T\left(x, a y_{1}-b y_{2}, z\right)-2 a^{2} T\left(x, y_{1}, z\right)-2 b^{2} T\left(x, y_{2}, z\right)\right\| \\
&=\lim _{n \rightarrow \infty}\left|a^{-7 s n}\right| \| f\left(a^{s n} x, a^{s n} a y_{1}+a^{s n} b y_{2}, a^{s n} z\right)+f\left(a^{s n} x, a^{s n} a y_{1}-a^{s n} b y_{2}, a^{s n} z\right) \\
& \quad-2 a^{2} f\left(a^{s n} x, a^{s n} y_{1}, a^{s n} z\right)-2 b^{2} f\left(a^{s n} x, a^{s n} y_{2}, a^{s n} z\right) \| \\
& \leq \lim _{n \rightarrow \infty}\left|a^{-7 s n}\right| \phi_{2}\left(a^{s n} x, a^{s n} y_{1}, a^{s n} y_{2}, a^{s n} z\right)=0, \\
& \| T\left(x, y, a z_{1}+b z_{2}\right)+T\left(x, y, a z_{1}-b z_{2}\right)-a^{2} b^{2}\left(T\left(x, y, z_{1}+z_{2}\right)+T\left(x, y, z_{1}-z_{2}\right)\right) \\
& \quad-2 a^{2}\left(a^{2}-b^{2}\right) T\left(x, y, z_{1}\right)+2 b^{2}\left(a^{2}-b^{2}\right) T\left(x, y, z_{2}\right) \| \\
&= \lim _{n \rightarrow \infty}\left|a^{-7 s n}\right| \| f\left(a^{s n} x, a^{s n} y, a^{s n} a z_{1}+a^{s n} b z_{2}\right)+f\left(a^{s n} x, a^{s n} y, a^{s n} a z_{1}-a^{s n} b z_{2}\right) \\
&-a^{2} b^{2}\left(f\left(a^{s n} x, a^{s n} y, a^{s n} z_{1}+a^{s n} z_{2}\right)-f\left(a^{s n} x, a^{s n} y, a^{s n} z_{1}-a^{s n} z_{2}\right)\right) \\
&-2 a^{2}\left(a^{2}-b^{2}\right) f\left(a^{s n} x, a^{s n} y, a^{s n} z_{1}\right)+2 b^{2}\left(a^{2}-b^{2}\right) f\left(a^{s n} x, a^{s n} y, a^{s n} z_{2}\right) \| \\
& \leq \lim _{n \rightarrow \infty}\left|a^{-7 s n}\right| \phi_{3}\left(a^{s n} x, a^{s n} y, a^{s n} z_{1}, a^{s n} z_{2}\right)=0
\end{aligned}
$$

for all $x_{1}, x_{2}, y_{1}, y_{2}, z_{1}, z_{2}, x, y, z \in X$. It follows from (2.15), (2.16) and (2.17) that $T$ satisfies (1.8). That is, $T$ is heptic.

By Theorem 1.1, we obtain that

$$
d(f, T) \leq \frac{1}{1-L} d(f, J f) \leq \frac{1}{1-L}
$$

which implies the inequality (2.6). Since $T$ is the unique fixed point of $J$ in the set $\Omega=\{g \in$ $S: d(f, g)<\infty\}, T$ is the unique mapping satisfying (2.6).

Remark 2.2 Let $X$ be a normed space and let $Y$ be a Banach space in Theorem 2.1. Using the direct method, one can show that there exists a unique heptic mapping $T: X \times X \times$ $X \rightarrow Y$ satisfying (1.8) and

$$
\|f(x, y, z)-T(x, y, z)\| \leq \frac{1}{2|a|} \tilde{\phi}_{1}(x, 0, y, z)+\frac{1}{2|a|^{3}} \tilde{\phi}_{2}(x, y, 0, z)+\frac{1}{2|a|^{7}} \tilde{\phi}_{3}(x, y, z, 0)
$$


for all $x, y, z \in X$, where we assume that

$$
\begin{aligned}
& \tilde{\phi}_{1}(x, 0, y, z):=\sum_{i=\frac{1-s}{2}}^{\infty} \frac{1}{a^{7 s i}} \phi_{1}\left(a^{s i} x, 0, a^{s i} y, a^{s i} z\right)<\infty, \\
& \tilde{\phi}_{2}(x, y, 0, z):=\sum_{i=\frac{1-s}{2}}^{\infty} \frac{1}{a^{7 s i}} \phi_{2}\left(a^{1+s i} x, a^{s i} y, 0, a^{s i} z\right)<\infty, \\
& \tilde{\phi}_{3}(x, y, z, 0):=\sum_{i=\frac{1-s}{2}}^{\infty} \frac{1}{a^{7 s i}} \phi_{3}\left(a^{1+s i} x, a^{1+s i} y, a^{s i} z, 0\right)<\infty .
\end{aligned}
$$

It is easy to show that the approximation in non-Archimedean spaces (inequality (2.6)) is better than the approximation in (Archimedean) normed spaces (inequality (2.18)).

The following corollary in the (Archimedean) normed spaces by the direct method is valid.

Corollary 2.3 Let $s \in\{-1,1\}$ be fixed and $\theta, r>0$ be real numbers such that $r s<7 s$, and let $X, Y$ be a normed space and a Banach space, respectively. Suppose that a mapping $f: X \times X \times X \rightarrow Y$ satisfies $f(x, 0, z)=f(x, y, 0)=0$ and

$$
\begin{aligned}
& \left\|f\left(a x_{1}+b x_{2}, y, z\right)+f\left(a x_{1}-b x_{2}, y, z\right)-2 a f\left(x_{1}, y, z\right)\right\| \leq \theta\left(\left\|x_{1}\right\|^{r}+\left\|x_{2}\right\|^{r}+\|y\|^{r}+\|z\|^{r}\right), \\
& \left\|f\left(x, a y_{1}+b y_{2}, z\right)+f\left(x, a y_{1}-b y_{2}, z\right)-2 a^{2} f\left(x, y_{1}, z\right)-2 b^{2} f\left(x, y_{2}, z\right)\right\| \\
& \quad \leq \theta\left(\|x\|^{r}+\left\|y_{1}\right\|^{r}+\left\|y_{2}\right\|^{r}+\|z\|^{r}\right), \\
& \| f\left(x, y, a z_{1}+b z_{2}\right)+f\left(x, y, a z_{1}-b z_{2}\right)-a^{2} b^{2}\left(f\left(x, y, z_{1}+z_{2}\right)+f\left(x, y, z_{1}-z_{2}\right)\right) \\
& \quad-2 a^{2}\left(a^{2}-b^{2}\right) f\left(x, y, z_{1}\right)+2 b^{2}\left(a^{2}-b^{2}\right) f\left(x, y, z_{2}\right) \| \leq \theta\left(\|x\|^{r}+\|y\|^{r}+\left\|z_{1}\right\|^{r}+\left\|z_{2}\right\|^{r}\right)
\end{aligned}
$$

for all $x, y, z, x_{1}, x_{2}, y_{1}, y_{2}, z_{1}, z_{2} \in X$. Then there exists a unique heptic mapping $T: X \times X \times$ $X \rightarrow Y$ satisfying (1.8) and a constant $M>0$ such that

$$
\|f(x, y, z)-T(x, y, z)\| \leq M\left(\|x\|^{r}+\|y\|^{r}+\|z\|^{r}\right)
$$

for all $x, y, z \in X$.

The following example shows that the previous corollary is not valid in non-Archimedean spaces.

Example 2.4 The most important examples of non-Archimedean spaces are $p$-adic numbers. A key property of $p$-adic numbers is that they do not satisfy the Archimedean axiom:

For all $x$ and $y>0$, there exists an integer $\mathrm{n}$ such that $x<n y$.

Let $p$ be a prime number. For any nonzero rational number $x$, there exists a unique integer $r \in \mathbb{Z}$ such that $x=\frac{a}{b} p^{r}$, where $a$ and $b$ are integers not divisible by $p$. Then $|x|_{p}:=p^{-r}$ defines a non-Archimedean norm on $\mathbb{Q}$ (we put $|0|_{p}=0$ ). The completion of $\mathbb{Q}$ with respect to $|\cdot|$ is denoted by $\mathbb{Q}_{p}$ and is called a $p$-adic number field. Note that if $p \geq 3$, then $\left|2^{n}\right|=1$ for each integer $n$. 
Let $X=Y=\mathbb{Q}_{p}$ for a prime number $p>3$ and define $f: X \times X \times X \rightarrow Y$ by $f(x, y, z)=x y z$. Then we have

$$
\left|f\left(2 x_{1}+x_{2}, y, z\right)+f\left(2 x_{1}-x_{2}, y, z\right)-4 f\left(x_{1}, y, z\right)\right|_{p}=|0|_{p}=0 \leq\left|x_{1}\right|_{p}+\left|x_{2}\right|_{p}+|y|_{p}+|z|_{p}
$$

and

$$
\begin{aligned}
& \left|f\left(x, 2 y_{1}+y_{2}, z\right)+f\left(x, 2 y_{1}-y_{2}, z\right)-8 f\left(x, y_{1}, z\right)-2 f\left(x, y_{2}, z\right)\right|_{p} \\
& \quad=\left|-4 y_{1}-2 y_{2}\right|_{p} \leq \max \left\{\left|-4 y_{1}\right|_{p},\left|-2 y_{2}\right|_{p}\right\} \\
& \quad \leq \max \left\{\left|y_{1}\right|_{p},\left|y_{2}\right|_{p}\right\} \leq|x|_{p}+\left|y_{1}\right|_{p}+\left|y_{2}\right|_{p}+|z|_{p}
\end{aligned}
$$

and

$$
\begin{aligned}
\mid f\left(x, y, 2 z_{1}+z_{2}\right)+f\left(x, y, 2 z_{1}-z_{2}\right)-4\left(f\left(x, y, z_{1}+z_{2}\right)+f\left(x, y, z_{1}-z_{2}\right)\right) & \\
& -24 f\left(x, y, z_{1}\right)+\left.6 f\left(x, y, z_{2}\right)\right|_{p} \\
= & \left|-24 z_{1}+6 z_{2}\right|_{p} \leq \max \left\{\left|-24 z_{1}\right|_{p},\left|6 z_{2}\right|_{p}\right\} \\
\leq & \max \left\{\left|z_{1}\right|_{p},\left|z_{2}\right|_{p}\right\} \leq|x|_{p}+|y|_{p}+\left|z_{1}\right|_{p}+\left|z_{2}\right|_{p} .
\end{aligned}
$$

But for each natural number $n$, we have

$$
\begin{aligned}
& \left|2^{-7 s(n-1)} f\left(2^{s(n-1)} x_{1}, 2^{s(n-1)} x_{2}, 2^{s(n-1)} x_{3}\right)-2^{-7 s n} f\left(2^{s n} x_{1}, 2^{s n} x_{2}, 2^{s n} x_{3}\right)\right|_{p} \\
& \quad=\left|2^{-4 s(n-1)} x_{1} x_{2} x_{3}-2^{-4 s n} x_{1} x_{2} x_{3}\right|_{p} \\
& \quad \leq \max \left\{\left|2^{-4 s(n-1)} x_{1} x_{2} x_{3}\right|_{p},\left|-2^{-4 s n} x_{1} x_{2} x_{3}\right|_{p}\right\}=\left|x_{1} x_{2} x_{3}\right|_{p} .
\end{aligned}
$$

Hence, for each $x_{1}, x_{2}, x_{3} \neq 0,\left\{2^{-7 s n} f\left(2^{s n} x_{1}, 2^{s n} x_{2}, 2^{s n} x_{3}\right)\right\}$ is not convergent.

\section{Competing interests}

The authors declare that they have no competing interests.

\section{Authors' contributions}

All authors conceived of the study, participated in its design and coordination, drafted the manuscript, participated in the sequence alignment, and read and approved the final manuscript.

\section{Author details}

'Department of Mathematics, Research Institute for Natural Sciences, Hanyang University, Seoul, 133-791, Korea. ${ }^{2}$ Department of Mathematics, Iran University of Science and Technology, Narmak, Tehran, Iran. ${ }^{3}$ Department of Mathematics, Semnan University, Semnan, Iran. ${ }^{4}$ Department of Mathematics, Faculty of Mathematical Sciences, University of Mohaghegh Ardabili, Ardabil, 56199-11367, Iran.

\section{Received: 3 December 2012 Accepted: 23 May 2013 Published: 12 July 2013}

\section{References}

1. Ulam, SM: Problems in Modern Mathematics. Science Editions, chapter VI. Wiley, New York (1964)

2. Hyers, DH: On the stability of the linear functional equation. Proc. Natl. Acad. Sci. USA 27, 222-224 (1941)

3. Aoki, T: On the stability of the linear transformation in Banach spaces. J. Math. Soc. Jpn. 2, 64-66 (1950)

4. Rassias, TM: On the stability of the linear mapping in Banach spaces. Proc. Am. Math. Soc. 72, 297-300 (1978)

5. Găvruta, P: A generalization of the Hyers-Ulam-Rassias stability of approximately additive mappings. J. Math. Anal. Appl. 184, 431-436 (1994)

6. Radu, V: The fixed point alternative and the stability of functional equations. Fixed Point Theory 4, 91-96 (2003) 
7. Cădariu, L, Radu, V: On the stability of the Cauchy functional equation: a fixed point approach. Grazer Math. Ber. 346, 43-52 (2004)

8. Cădariu, L, Radu, V: Fixed points and the stability of Jensen's functional equation. J. Inequal. Pure Appl. Math. 4, Article ID 4 (2003)

9. Margolis, B, Diaz, JB: A fixed point theorem of the alternative for contractions on the generalized complete metric space. Bull. Am. Math. Soc. 126, 305-309 (1968)

10. Hensel, K: Über eine neue Begründung der Theorie der algebraischen Zahlen. Jahresber. Dtsch. Math.-Ver. 6, 83-88 (1897)

11. Khrennikov, A: Non-Archimedean Analysis: Quantum Paradoxes, Dynamical Systems and Biological Models. Kluwer Academic, Dordrecht (1997)

12. Arriola, LM, Beyer, WA: Stability of the Cauchy functional equation over $p$-adic fields. Real Anal. Exch. 31, 125-132 (2005/2006)

13. Narici, L, Beckenstein, E: Strange terrain-non-Archimedean spaces. Am. Math. Mon. 88, 667-676 (1981)

14. Park, C, Boo, D, Rassias, TM: Approximately additive mappings over $p$-adic fields. J. Chungcheong Math. Soc. 21, 1-14 (2008)

15. Vladimirov, VS, Volovich, IV, Zelenov, El: p-Adic Analysis and Mathematical Physics. World Scientific, Singapore (1994)

16. Khodaei, $\mathrm{H}$, Rassias, TM: Approximately generalized additive functions in several variables. Int. J. Nonlinear Anal. Appl. 1, 22-41 (2010)

17. Aczel, J, Dhombres, J: Functional Equations in Several Variables. Cambridge University Press, Cambridge (1989)

18. Kannappan, P: Quadratic functional equation and inner product spaces. Results Math. 27, 368-372 (1995)

19. Skof, F: Local properties and approximations of operators. Rend. Semin. Mat. Fis. Milano 53, 113-129 (1983)

20. Czerwik, S: On the stability of the quadratic mapping in normed spaces. Abh. Math. Semin. Univ. Hamb. 62, 59-64 (1992)

21. Eshaghi Gordji, M, Khodabakhsh, R, Jung, S, Khodaei, H: AQCQ-functional equation in non-Archimedean normed spaces. Abstr. Appl. Anal. 2010, Article ID 741942 (2010)

22. Lee, S, Im, S, Hawng, I: Quartic functional equation. J. Math. Anal. Appl. 307, 387-394 (2005)

23. Kang, D: On the stability of generalized quartic mappings in quasi- $\beta$-normed spaces. J. Inequal. Appl. 2010, Article ID $198098(2010)$

24. Chung, JK, Sahoo, PK: On the general solution of a quartic functional equation. Bull. Korean Math. Soc. 40, 565-576 (2003)

25. Kim, H: On the stability problem for a mixed type of quartic and quadratic functional equation. J. Math. Anal. Appl. 324, 358-372 (2006)

26. Lee, Y, Chung, S: Stability of quartic functional equations in the spaces of generalized functions. Adv. Differ. Equ. 2009, Article ID 838347 (2009)

27. Najati, A: On the stability of a quartic functional equation. J. Math. Anal. Appl. 340, 569-574 (2008)

28. Park, W, Bae, J: On a bi-quadratic functional equation and its stability. Nonlinear Anal. TMA 62, 643-654 (2005)

29. Eshaghi Gordji, M: Stability of a functional equation deriving from quartic and additive functions. Bull. Korean Math. Soc. 47, 491-502 (2010)

30. Eshaghi Gordji, M: Stability of an additive-quadratic functional equation of two variables in F-spaces. J. Nonlinear Sci. Appl. 2, 251-259 (2009)

31. Eshaghi Gordji, M, Abbaszadeh, S, Park, C: On the stability of generalized mixed type quadratic and quartic functional equation in quasi-Banach spaces. J. Inequal. Appl. 2009, Article ID 153084 (2009)

32. Eshaghi Gordji, M, Alizadeh, Z, Cho, Y, Khodaei, H: On approximate $C^{*}$-ternary $m$-homomorphisms: a fixed point approach. Fixed Point Theory Appl. 2011, Article ID 454093 (2011)

33. Eshaghi Gordji, M, Bavand Savadkouhi, M: On approximate cubic homomorphisms. Adv. Differ. Equ. 2009, Article ID $618463(2009)$

34. Eshaghi Gordji, M, Bodaghi, A: On the stability of quadratic double centralizers on Banach algebras. J. Comput. Anal. Appl. 13, 724-729 (2011)

35. Eshaghi Gordji, M, Bodaghi, A, Alias, IA: On the stability of quadratic double centralizers and quadratic multipliers: a fixed point approach. J. Inequal. Appl. 2011, Article ID 957541 (2011)

36. Eshaghi Gordji, M, Farokhzad Rostami, R, Hosseinioun, SAR: Nearly higher derivations in unital C*-algebras. J. Comput. Anal. Appl. 13, 734-742 (2011)

37. Eshaghi Gordji, M, Ghaemi, MB, Majani, H, Park, C: Generalized Ulam-Hyers stability of Jensen functional equation in Šrstnev PN spaces. J. Inequal. Appl. 2010, Article ID 868193 (2010)

38. Eshaghi Gordji, M, Kaboli-Gharetapeh, S, Park, C, Zolfaghri, S: Stability of an additive-cubic-quartic functional equation. Adv. Differ. Equ. 2009, Article ID 395693 (2009)

39. Eshaghi Gordji, M, Kaboli Gharetapeh, S, Rassias, JM, Zolfaghari, S: Solution and stability of a mixed type additive, quadratic and cubic functional equation. Adv. Differ. Equ. 2009, Article ID 826130 (2009)

40. Eshaghi Gordji, M, Karimi, T, Kaboli Gharetapeh, S: Approximately n-Jordan homomorphisms on Banach algebras, J. Inequal. Appl. 2009, Article ID 870843 (2009)

41. Eshaghi Gordji, M, Savadkouhi, MB: Stability of a mixed type cubic and quartic functional equations in random normed spaces. J. Inequal. Appl. 2009, Article ID 527462 (2009)

42. Eshaghi Gordji, M, Savadkouhi, MB, Park, C: Quadratic-quartic functional equations in RN-spaces. J. Inequal. Appl. 2009, Article ID 868423 (2009)

43. Ebadian, A, Najati, A, Eshaghi Gordji, M: On approximate additive-quartic and quadratic-cubic functional equations in two variables on Abelian groups. Results Math. 58, 39-53 (2010)

44. Hadžić, O, Pap, E, Radu, V: Generalized contraction mapping principles in probabilistic metric spaces. Acta Math. Hung. 101, 131-148 (2003) 